\title{
THE CORRELATION ANALYSIS OF SUBSIDENCE MONITORING BY D-INSAR AND THE CHANGE OF URBAN CONSTRUCTION LAND
}

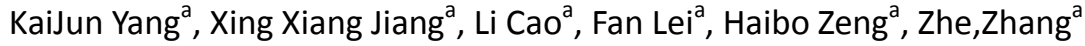 \\ Hunan land and Resources Planning Institute, Hunan, People's Republic of China \\ apple_hacker@163.com
}

KEY WORDS: DInSAR， subsidence, interferometry, correlation ABSTRACT:

The change of urban construction land affect the subsidence directly or indirectly, the method of D-InSAR has centimeter level or even millimeter accuracy that can provide a reliable and accurate data for the research of correlation analysis of subsidence monitoring by D-InSAR and the change of urban construction land. This article takes Guiyang, Nanning city as example, using 3m level TerraSAR data to construct the Subsidence model by interferometric measurement, then compared with the Chinese national land use change remote sensing survey database at the same measure time to have a correlation analysis GIS research between subsidence and the change of urban construction land. The results shows that the integral correlation coefficient achieved 0.78 between subsidence and the change of urban construction land, the major construction area and the high density construction area are with severe land subsidence. In addition, the correlation coefficient increased from the main city to the suburbs, indicates that some of the main city causes permanent settlement and is difficult to recover. It also shows that some area subsidence caused by long-term mining or other natural factors has no strong correlation with the change of urban construction land, therefore, the results of D-InSAR subsidence monitoring have a reaction on urban construction planning, guiding urban planning to high stability, low settlement area.

\section{INTRODUCTION}

The routine monitoring ways of ground subsidence is mainly by levelling, GPS measurement, stratified standard measurement and so on. These ground measurements can only measure subsidence information at sparse points on the ground, lack of spatial density, difficult to cover large areas and higher cost. Satellite Synthetic Aperture Radar Interferometry(INSAR) is internationally recognized as the most effective and advanced ways of ground subsidence monitoring. At present, TerraSAR, Sentinel-1, EnviSat satellites and GF3 provide reliable protection data sources for large-scale urban settlement monitoring. Many scholars in the world have done a lot of research by INSAR technology. However, due to the lack of official urban land change data, the correlation analysis between urban land change data and D-InSAR surface subsidence still in a relatively vacant state, cannot be used for the city planning, stable development pattern to provide a scientific basis for analysis of relevance.
Based on this situation, this paper measure the surface subsidence of Nanning area by using the TerraSAR-X data from January 2016 to August 2016. Then made a correlation study combined with the land and resources data by remote sensing of People's Republic of China Ministry of Land and Resources. Based on the correlation results, the reason of the settlement were analysed, and some suggestions are put forward for the urban settlement control policy and the development pattern.

\section{INSAR SETTLEMENT MONITORING TECHNOLOGY}

INSAR is an active remote sensing method by using synthetic aperture radar on satellites, and it is a method of extracting the three-dimensional information and changing information of the surface by using the phase information extracted from the composite aperture radar data. The INSAR obtains a pair of complex images of the same landscape by observing the two antennas simultaneously (monorail mode), or two near-parallel observations (repetitive 
orbital mode). Due to the geometric relationship between the target and the two antenna positions, a phase difference is generated on the complex image to form an interference pattern. The interference pattern contains accurate information about the difference between the point up the slope and the position of the two antennas.

The geometrical relative relationship between the satellite orbit and the ground target of the repeated orbital interference is shown as below:

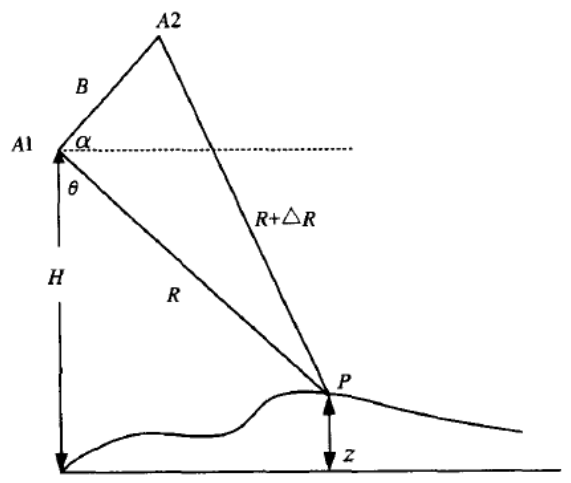

Figure 1. Figure of "Double track" method

In the figure, $\mathrm{A} 1$ and $\mathrm{A} 2$ represent the positions of the two antennas respectively; B represent the distance between the antennas base line; the angle between the baseline and the horizontal direction is $\alpha$; $\mathrm{H}$ is the height of the platform; $\theta$ is the reference line angle of the first sub antenna, terrain elevation is $\mathrm{z}$.

The interference phase at point $\mathrm{P}$ is:

$\phi=-\frac{4 \pi}{\lambda} B \sin (\theta-\alpha) ; \quad \mathrm{z}=H-R \cos \theta$

Differential interferometry (DINSAR) imaging geometry (in the case of a three-track method) is shown as below, $\Delta \mathrm{Rd}$ is the line of sight to the deformed. A1 and A2 are the two satellite locations where the satellite is imaged in the same area. The first SAR image is obtained before the surface deformation occurs, and the second SAR image is obtained after the surface deformation. The phase of the interference pattern formed by the two SAR images both contains the terrain information of the area and the observation during the deformation of the surface information.

$$
\phi_{1}=-\frac{4 \pi}{\lambda} B \sin \left(\theta_{1}-\alpha_{p}\right)-\frac{4 \pi}{\lambda} \Delta R_{d}
$$

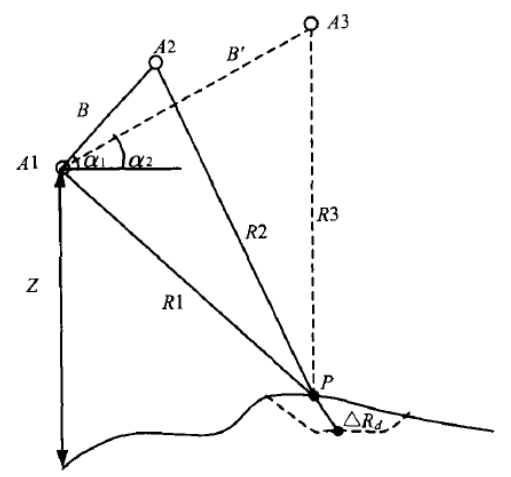

Figure 2. Figure of "Three-track" method

It is necessary to eliminate the terrain information of the area before get the deformation information of the surface, we then use the third SAR image to eliminate the impact of terrain by interference methods. It is known as the "three-track method".

The third image is acquired before the deformation occurs, the interference pattern formed by the third image and the first image, and the interference phase contains only terrain information.

$$
\phi_{2} \approx-\frac{4 \pi}{\lambda} B^{\prime} \sin \left(\theta_{2}-\alpha_{2}\right)
$$

The phase caused by the line of sight to the deformation $\Delta \mathrm{Rd}$ is:

$$
\phi_{\mathrm{d}}=-\frac{4 \pi}{\lambda} \Delta R_{d}
$$

The amount of the left side of the above formula can be calculated from the phase and orbital parameters of the interference pattern, and then the line of sight of each point of view to the deformation variable $\Delta \mathrm{Rd}$ is obtained, then the entire area of the surface deformation is calculated.

\section{EXPERIMENTAL STUDY}

\subsection{General Situation and Data Preparation}

Nanning City is located in the southwest of China, is the capital of Guangxi Province, it is the northern Gulf Economic Zone and the core cities in southern China. The crust is relatively stable in the area, but there is a 
lot of groundwater exploitation in the old city. The crust is relatively stable in the area, but there is a lot of groundwater exploitation in the old city.

All-weather remote sensing monitoring is a project carried out by the Ministry of Land and Resources to monitor annual changes in urban construction data changes, the development of city border. Aiming to analyse the technical results of the land use credit file by analysing the monitoring results of each city. In the case of SAR orthographic imaging monitoring in Nanning area, the two time nodes monitoring are distributed in January 2016 and August 2016, and more than 500 urban construction spot are extracted through the refinement of human-computer interaction work.

In order to analyse the correlation between the data results, the TerraSAR-X monitoring data with the resolution of $3 \mathrm{~m}$ at same period with the monitoring spot were selected. The incident angle was 33 degrees, and the experiment was selected The Nanning area in January and August, a total of six high-repeat coverage of the same track TerraSAR data for differential interference measurement.

\subsection{Data Processing}

Firstly, estimate the baseline image of the pairs SAR image to be processed, he spatial baseline length of the three pairs of images is $24 \mathrm{~m}, 57 \mathrm{~m}, 46 \mathrm{~m}$, the time baseline length is 204 days, 211 days, 213 days. The SRTM 90m grid is selected as the base DEM data. The specific solution flow is Interferogram Generation-> Adaptive Filter and Coherenace Generation-> Phase Unwrapping-> GCP Selection-> Refinement and Reflattening-> Phase Dispalcement and Conversion and Geocoding.

The Principal Parameters is as follows:

Range Looks:1,

Azimuth Looks:1,

Grid Size for Suggested Looks:3m,

Filter Method:Goldstein,
Unwrapping Method: Minimum Cost Flow,

Unwrapping Coherence Threshold:0.20,

Refinement Res Phase Poly Degree:3

In order to eliminate the influence of orbit error, the DEM registration strategy is taken into account in the spectral offset calculation to ensure that the local terrain information.

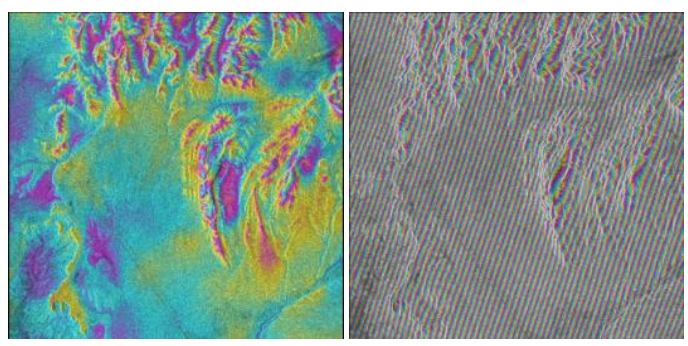

Figure 3. Interference fringe results

\section{RESULT ANALYSIS}

The experiment got the settlement results of Nanning city (as shown below), The maximum settlement of the area reached $14.5 \mathrm{~mm}$, and the maximum lifting of the surface reached $16.2 \mathrm{~mm}$, with an average value of $0.62 \mathrm{~mm}$, and the standard deviation is $3.94 \mathrm{~mm}$, It is found that there is a large settling funnel in the northwest of the region which is located in Xixiangtang area of Nanning city, and the average settlement of the core area is $10.6 \mathrm{~mm}$. There is a significant uplift in the middle and southwest of the study area.

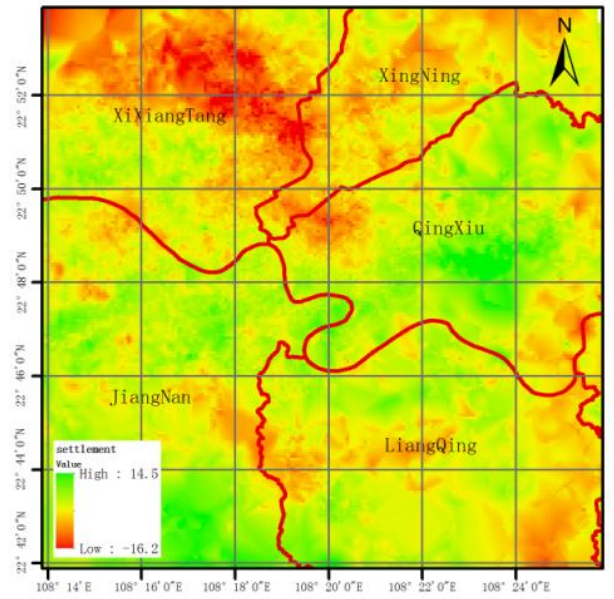

Figure 4. Nanning urban settlement results 
In order to study the correlation between the all-weather remote sensing monitoring plots and the regional subsidence, the spot of new construction and the subsidence were superimposed (as shown below), In the figure, the fragmented spots shows the new construction land data of the all-weather change survey, and it is found that the core construction area appears positively correlated with the degree of settlement, especially like XiXiangTang District in the northwest and LiangQing District in the south, Both of the settlement funnel with a higher higher construction intensity. In order to express the correlation between the data objectively, the mean settlement value covered by the spot represent the degree of subsidence of the plots, the density of the plots in the radius of $500 \mathrm{~m}$ near the plots is taken as the concentration of the plots, then obtain the correlation between the degree of subsidence and the degree of plot concentration.

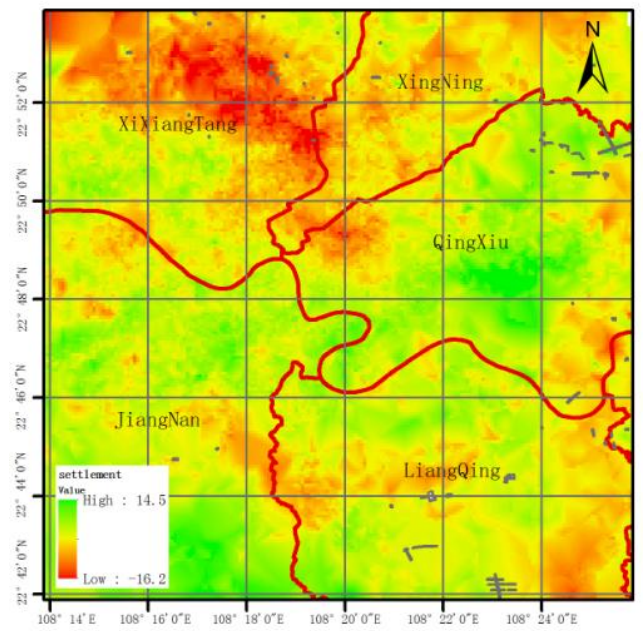

Figure 5.Construction monitoring spots and settlement result

the degree of plot settlement:

$$
\mathrm{D}_{s}=\frac{\sum_{i=0}^{n} s_{i}}{n}
$$

$\mathrm{S}_{\mathrm{i}}$ represents the settlement value of a pixel in the area covered by the spot, $\mathrm{n}$ indicates the number of pixels within the spot。

The degree of plot concentration:

$$
\mathrm{D}_{\mathrm{f}}=\frac{\sum_{i=0}^{k} A_{i}}{A r e}
$$

Ai represents the area of the spot, $\mathrm{k}$ represents the number of plots in the radius range of $500 \mathrm{~m}$, Area represents the area of the circle with a radius of 500 m.

The correlation index:

$\mathrm{R}\left(D_{s}, D_{f}\right)=\frac{\operatorname{cov}\left(D_{s}, D_{f}\right)}{\sqrt{D\left(D_{s}\right) \cdot D\left(D_{f}\right)}}=\frac{\sum_{i=1}^{n}\left(D_{s i}-\bar{D}_{s}\right)\left(D_{f i}-\bar{D}_{f}\right)}{\sqrt{\sum_{i=1}^{n}\left(D_{s i}-\bar{D}_{s}\right) \sum_{i=1}^{2}\left(D_{f i}-\bar{D}_{f}\right)^{2}}}$

Through the above calculation method, the correlation between the the degree of plot settlement and the degree of concentration is 0.78 , which is close to the high correlation.

\begin{tabular}{|l|c|}
\hline District & correlation \\
\hline & \\
XiXiangTang & 0.87 \\
XingNing & 0.72 \\
QingXiu & 0.69 \\
JiangNan & 0.76 \\
LiangQing & 0.71 \\
Total & 0.78 \\
\hline
\end{tabular}

Table 1. Distribution of correlation index

It is also found that Xixiangtang District is the old city of Nanning City, and occurred many cracking events by settlement, this place is also one of the most serious areas of groundwater exploitation. High positive correlation shows that the urban planning decision-making does not take the surface subsidence factor into consideration. There will be a hidden danger of settlement prevention and control work with sustained high-intensity construction. The city's urban development should focus on the smaller settlement of the study results, rather than strengthen the construction in the settlement funnel, In addition, governance work should be taken into account of two larger sedimentation funnel areas in XiXiangTang District and LiangQing District, forming a scientific planning and construction distribution. 


\section{CONCLUSIONS AND PROSPECTS}

In this experiment, the settlement results of January 2016 to August 2016 in Nanning area were calculated by DInSAR. Two large settling funnels were found in the northwest and southern regions, and there is a high positive correlation with all-weather city change monitoring spots, indicates that the planning and construction work in the region is towards to the wrong spatial orientation. We will explore the reasons for the formation of sedimentation funnel in the future, control the settlement from the source.

\section{REFERENCES}

Rodriguez E, Martin JM . Theory and Design of Interferometric Synthetic Aperture Radars[J]. IEEProc.-F, 1992, 139(2): 147- 159.

Hebble White B , Waddington A,Wood JH .Regional Horizontal Surface Displacements Due to Mining Beneath Severe Surface To-pography [A]. 19th Conference on Ground Control in Mining [C].

Morgan town, West Virginia, USA, 2000.

Massonnet D, Rossi M. The Displacement Field of the Landers Earthquake Mapped by Radar Interferometry

[J]. Nature, 1993, 364(6 433): 138-142

Amelung F, Jónssen S, Zebker H, et al. Wide-spread Uplift and Trap Door Faulting on Gal Pagos

Volcanoes Observed with Radar Interferometry [J].

Nature,2000,407(6 807):993-996
Hanssen R H. Radar Interferometer-Data Interpretation and Error Analysis[ M]. Netherlands:

Kluwer Academic Publishers, 2001

LIUG.X.,CHENY.Q.,DING X.L ., etal., 2002.

Monitoring Ground Settlement in HongKong with Satellite SAR Interferometry, FIG XXII International Congress, Washington,D.C,USA, $19 \sim 26$.

Zhao Chaoying,Ding Xiaoli,Zhang Qin,et al.Monitoring of Recent Land Subsidence and Ground Fissures in Xi' an with SAR Interferometry[C].ISPRS, Beijing,2008.

Hooper A, Zebker H A.Phase Unwrapping in Three Dimensions with Application to InSAR Time Series[J].J Opt Soc Am.A: Opt.Image Sci. Vis.,2010,24(9):2 737-2 747

Li Z,Fielding E J,Cross P,et al.Interferometric Synthetic Aperture Radar Atmospheric Correction: GPS Topography-dependent Turbulence Model[J]. J Geophys Res,2006,111(B2):B02404

GE L,CHANG H C,RIZOS C. Mine subsidence monitoring using multi-source satellite SAR images[J].Photogrammetric Engineering and Remote Sensing,2007,73(3):259-266.

HAYMAN A,GE L,YAN Yueguan.Mapping accumulated mine subsidence using small stack of SAR differential interferograms in the Southern coalfield of New South Wales,Australia[J]. Engineering Geology,2010,115(1/2):1 